\title{
Collective Pitch Feedforward Control of Floating Wind Turbines Using Lidar
}

\author{
David Schlipf ${ }^{1 \& 2}$, Eric Simley ${ }^{2}$, Frank Lemmer ${ }^{1}$, Lucy Pao ${ }^{2}$, Po Wen Cheng ${ }^{1}$ \\ ${ }^{1}$ Stuttgart Wind Energy, Universität Stuttgart, Stuttgart, Germany \\ ${ }^{2}$ Department of Electrical, Computer, and Energy Engineering, University of Colorado, Boulder, Colorado, USA
}

\begin{abstract}
In this work a collective pitch feedforward controller for floating wind turbines is presented. The feedforward controller provides a pitch rate update to a conventional feedback controller based on a wind speed preview. The controller is designed similar to the one for onshore turbines, which has proven its capability to improve wind turbine control performance in field tests. In a first design step, perfect wind preview and a calm sea is assumed. Under these assumptions the feedforward controller is able to compensate almost perfectly the effect of changing wind speed to the rotor speed of a full nonlinear model over the entire full load region. In a second step, a nacelle-based lidar is simulated scanning the same wind field which is used also for the aero-hydro-servo-elastic simulation. With model-based wind field reconstruction methods, the rotor effective wind speed is estimated from the raw lidar data and is used in the feedforward controller after filtering out the uncorrelated frequencies. Simulation results show that even with a more realistic wind preview, the feedforward controller is able to significantly reduce rotor speed and power variations. Furthermore, structural loads on the tower, rotor shaft, and blades are decreased. A comparison to a theoretical investigation shows that the reduction in rotor speed regulation is close to the optimum.
\end{abstract}

\section{KEYWORDS}

floating wind turbine control; feedforward control; lidar.

\section{INTRODUCTION}

Floating wind turbines offer a promising solution for harvesting the immense potential of wind energy at deep water locations. New concepts have to be devised particularly for collective blade pitch control to regulate the rotor speed because of the known negative damping of the platform pitch motion at low frequencies introduced by the wind variations at these frequencies (Fleming et al., 2014). Based on van der Veen et al. (2012), there are mainly four possibilities to face this problem by feedback control: A straightforward approach is to lower the closed-loop bandwidth of the pitch controller under the platform pitch frequency as done by Jonkman (2007) and Larsen and Hanson (2007). The second possibility is to use acceleration measurements to damp the pitch motion (Lackner and Rotea, 2011). Another option is to add additional control inputs, such as the generator torque (Fischer, 2012), individual blade pitch (Namik and Stol, 2010) or active mass dampers (Lackner and Rotea, 2011). Finally, using a blade pitch to stall controller as mentioned by Larsen and Hanson (2007) would avoid the negative damping. Alternative methods become feasible with lidar remote sensing technology, where information about incoming wind speed changes can be made available ahead of time and thus can be used with model predictive (Schlipf et al., 2013b; Raach et al., 2014) or feedforward control algorithms.

This work presents a collective pitch feedforward controller for floating wind turbines. The approach is based on a controller which was developed for onshore wind turbines and successfully tested in various field campaigns by Schlipf et al. (2014) and Scholbrock et al. (2013). The presented approach divides the lidar-assisted control design into two steps: In the first step, the pure control problem is considered and perfect wind speed preview is assumed. The feedforward controller is designed based on the static, nonlinear pitch curve and is combined with the baseline feedback controller. In a simulation with a fully aero-hydro-servoelastic model assuming perfect wind preview, the feedforward controller is able to keep the rotor speed nearly unaffected by an extreme operating gust. This demonstrates robustness against model uncertainties. In the second step, the problem is extended to accommodate the limited preview information obtained by a lidar system. Conventional lidar systems are only able to measure the line-of-sight velocity at limited points in front of the turbine. Additionally, nacelle-based systems on floating wind turbines experience significant motions. As a result, only the low frequencies of the wind speed preview can be captured accurately. The controller is extended by adding lidar data processing and adaptive filtering stages. The extended controller is tested under realistic conditions using a turbulent wind field and a detailed lidar simulator. The obtained spectrum of the rotor speed is compared to the theoretical optimal spectrum that can be achieved with the used lidar setup. The optimal spectrum is calculated using a linearized turbine model and an analytic model of the correlation between the lidar measurements and the reaction of the wind turbine (Simley and Pao, 2013). 


\section{SIMULATION ENVIRONMENT}

For this study, a $5 \mathrm{MW}$ turbine on a spar-buoy is used (Jonkman, 2007) and implemented in the coupled aero-hydro-servo-elastic simulation tool FAST (Jonkman and Buhl, 2005). The controller is implemented in Matlab and coupled to FAST. The wind turbine experiences the disturbance of a turbulent three-dimensional wind field generated with TurbSim (Jonkman, 2009). The wind fields are loaded into Matlab and scanned during the simulations with a realistic lidar simulator. The wind turbine and the lidar simulator are described in the following subsections.

\section{Floating Wind Turbine}

The coupled FAST model for the floating offshore wind turbine (FOWT) system consists of a flexible multibody system, which experiences external forces from aerodynamics, hydrodynamics, and the mooring system. These are calculated in dedicated submodules of the code. The following 21 degrees of freedom (DOFs) are enabled in the simulations: platform translational (surge, sway, heave) and rotational (roll, pitch, yaw) DOFs, first and second flapwise and first edgewise blade modes, first and second fore-aft and side-to-side tower bending modes, rotor motion and drive train rotational-flexibility. The vector of these modes is denoted $q$ and its derivatives $\dot{q}$. A second-order linear model is added for the collective pitch actuator, resulting in a total of 22 DOFs. The hydrodynamic model is based on linear potential flow theory with the damping term of Morison's equation to account for viscous effects. The frequency-dependent solutions to the separated radiation and diffraction problem are solved in a preprocessing step by a hydrodynamic panel code. During the simulation, the pre-calculated fluid velocity and accelerations on several strips along the platform act as disturbance inputs to the hydrodynamic subsystem. In the aerodynamic subsystem, the disturbance inputs are the components of a turbulent threedimensional wind field on several grid points over the rotor disk. With these inputs, aerodynamic forces are calculated by applying BEM (Blade Element Momentum) theory. The floating spar-buoy is anchored over three slack mooring lines attached at the fairleads below the center of buoyancy. Horizontal and vertical forces at the fairleads are calculated by iteratively solving a quasi-static equation for a slack line, accounting for seabed friction.

The three translational platform DOFs are defined in the inertial coordinate system, denoted $\mathcal{I}$. Its origin is the mean sea level of the non-deflected FOWT $(q=\dot{q}=0)$. The turbulent wind fields are defined in the TurbSim coordinate system, which has the same orientation as the inertial coordinate system $\mathcal{I}$ and has its origin at the hub of the non-deflected FOWT. Thus, the wind field can be transformed to the $\mathcal{I}$ coordinate system by a simple translation of the TurbSim coordinate system.

\section{Lidar Simulator}

In this work, a nacelle-based scanning lidar system is simulated The simulator is based on (Schlipf et al., 2009) and is extended by including the platform motion to realistically reproduce lidar measurements from a floating wind turbine. The platform and turbine motion highly influence the lidar measurement, since the coordinates $\left[\begin{array}{lll}x_{i, \mathcal{L}} & y_{i, \mathcal{L}} & z_{i, \mathcal{L}}\end{array}\right]^{T}$ of the lidar measurements are designed in the lidar coordinate system $\mathcal{L}$, but change their locations in the inertial coordinate system $\mathcal{I}$ because of the system's motion.

In general, a lidar system is only able to measure the component

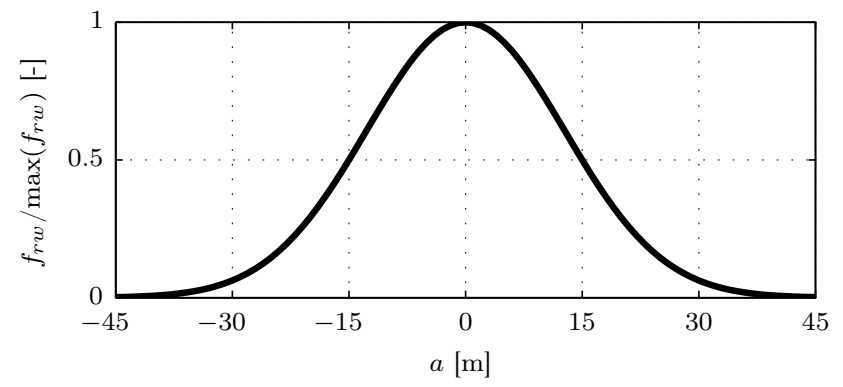

Figure 1: Normalized range weighting function for the used pulsed lidar system.

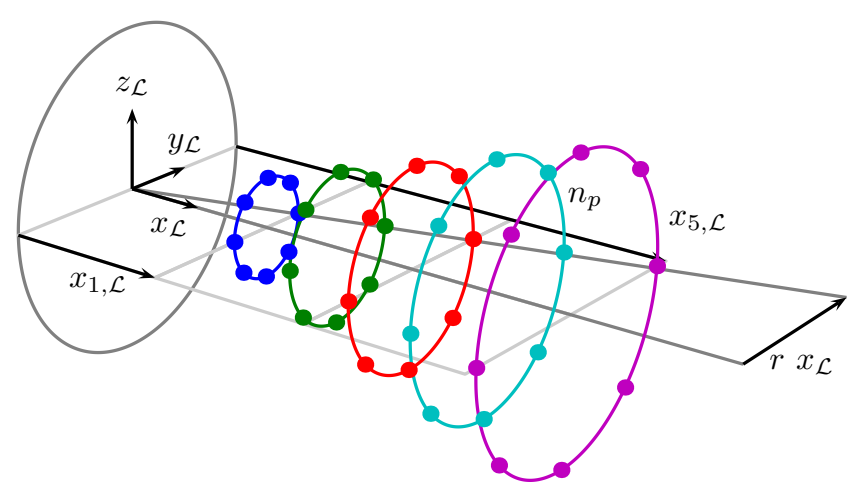

Figure 2: Optimized lidar scan trajectory.

of the wind vector in the laser beam direction. Therefore, the line-of-sight wind speed $v_{l o s P, i}$ measured by a stationary lidar system at point $i$ with coordinates $\left[x_{i, \mathcal{I}} y_{i, \mathcal{I}} z_{i, \mathcal{I}}\right]^{T}$ can be modeled by a projection of the wind vector $\left[\begin{array}{lll}u_{i, \mathcal{I}} & v_{i, \mathcal{I}} & w_{i, \mathcal{I}}\end{array}\right]^{T}$ at point $i$ on the normalized vector of the laser beam. This is mathematically equivalent to the scalar product of both vectors:

$$
v_{l o s P, i}=x_{n i, \mathcal{I}} u_{i, \mathcal{I}}+y_{n i, \mathcal{I}} v_{i, \mathcal{I}}+z_{n i, \mathcal{I}} w_{i, \mathcal{I}},
$$

where the normalized laser vector measuring at a distance $r_{i}$ from the lidar system is

$$
\left[\begin{array}{l}
x_{n i, \mathcal{I}} \\
y_{n i, \mathcal{I}} \\
z_{n i, \mathcal{I}}
\end{array}\right]=\frac{1}{r_{L i}}\left[\begin{array}{l}
x_{L, \mathcal{I}}-x_{i, \mathcal{I}} \\
y_{L, \mathcal{I}}-y_{i, \mathcal{I}} \\
z_{L, \mathcal{I}}-z_{i, \mathcal{I}}
\end{array}\right] \text { with } r_{L i}=\sqrt{x_{i, \mathcal{L}}^{2}+y_{i, \mathcal{L}}^{2}+z_{i, \mathcal{L}}^{2}}
$$

The position of the lidar system within the inertial coordinate system is defined by $\left[x_{L, \mathcal{I}} y_{L, \mathcal{I}} z_{L, \mathcal{I}}\right]^{T}$. However, real lidar systems measure within a probe volume. For pulsed lidar systems, this is due to the length of the emitted pulse (Cariou, 2013). If additionally the lidar system is not fixed in the inertial frame, but moving with the velocity $\left[\dot{x}_{L, \mathcal{I}} \dot{y}_{L, \mathcal{I}} \dot{z}_{L, \mathcal{I}}\right]^{T}$, Equation (1) can be adjusted as follows:

$$
\begin{aligned}
v_{l o s, i} & =\int_{-\infty}^{\infty}\left(x_{n i, \mathcal{I}}\left(u_{a i, \mathcal{I}}-\dot{x}_{L, \mathcal{I}}\right)+y_{n i, \mathcal{I}}\left(v_{a i, \mathcal{I}}-\dot{y}_{L, \mathcal{I}}\right)\right. \\
& \left.+z_{n i, \mathcal{I}}\left(w_{a i, \mathcal{I}}-\dot{z}_{L, \mathcal{I}}\right)\right) f_{r w}(a) \mathrm{d} a .
\end{aligned}
$$

The volume measurement is modeled by the range weighting function $f_{r w}$ depending on the distance $a$ to the measurement point. 

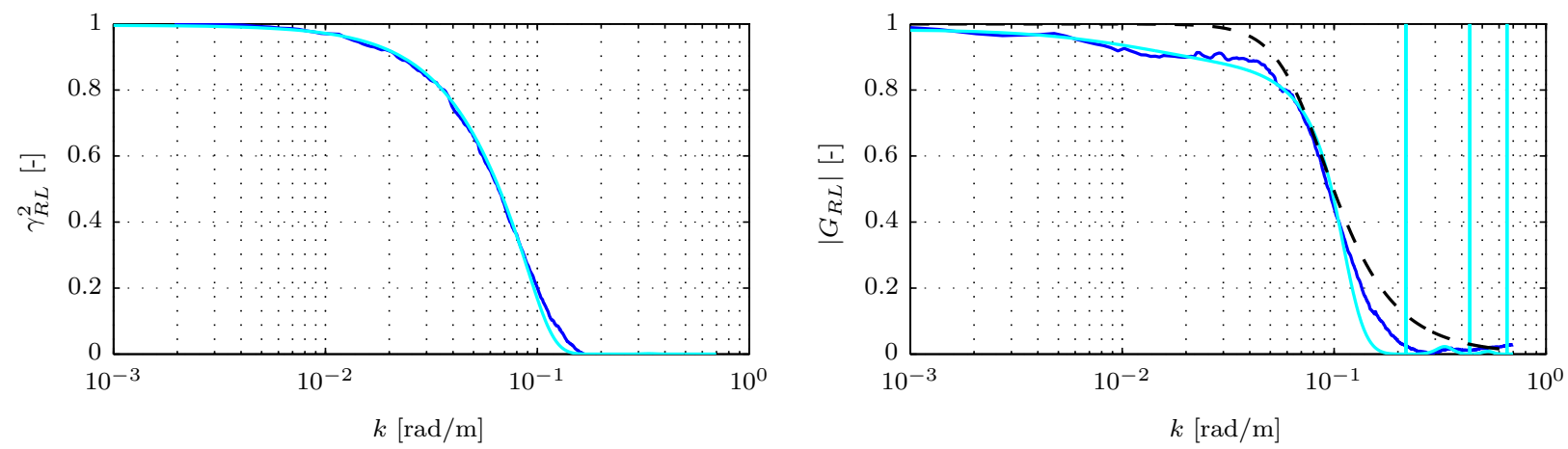

Figure 3: Coherence (left) and transfer function (right) between the lidar estimate of the rotor-effective wind speed and the real rotor-effective wind speed: based on the analytical model (-) and from simulation (-). Fitted filter (--).

For the pulsed lidar system considered in this work, a normalized Gaussian shape weighting function is used (see Figure 1), following Cariou (2013). The function is parameterized by a standard deviation $\sigma_{L}$ depending on the pulse width at half maximum of $W_{L}=30 \mathrm{~m}$ :

$$
f_{r w}(a)=\frac{1}{\sigma_{L} \sqrt{2 \pi}} \exp \left(-\frac{a^{2}}{2 \sigma_{L}^{2}}\right) \text { with } \sigma_{L}=\frac{W_{L}}{2 \sqrt{2 \ln 2}}
$$

The wind vector $\left[\begin{array}{lll}u_{a i, \mathcal{I}} & v_{a i, \mathcal{I}} & w_{a i, \mathcal{I}}\end{array}\right]^{T}$ is an evaluation of the wind field along the laser beam at

$$
\left[\begin{array}{l}
x_{a i, \mathcal{I}} \\
y_{a i, \mathcal{I}} \\
z_{a i, \mathcal{I}}
\end{array}\right]=\left[\begin{array}{l}
x_{i, \mathcal{I}} \\
y_{i, \mathcal{I}} \\
z_{i, \mathcal{I}}
\end{array}\right]+a\left[\begin{array}{l}
x_{n i, \mathcal{I}} \\
y_{n i, \mathcal{I}} \\
z_{n i, \mathcal{I}}
\end{array}\right]
$$

During the hydro-servo-aero-elastic simulations, the lidar simulator calculates the lidar position, velocity, and inclination based on the current values of all 6 platform modes and all 4 tower modes and their derivatives. The line-of-sight wind speeds $v_{l o s, i}$ are then calculated using Equations (2) to (5) and applying Taylor's Hypothesis of Frozen Turbulence (Taylor, 1938), which assumes that turbulent wind travels with the mean wind speed from the measurement location to the rotor.

For this work, the scan trajectory is optimized for the $5 \mathrm{MW}$ onshore reference wind turbine using the method presented in (Schlipf et al., 2013a). The optimal trajectory is found by calculating the coherence between the rotor-effective wind speed and its lidar estimate based on an analytic correlation model for several circular scan setups. The trajectory with the highest value of the coherence bandwidth is the circle with $n_{P}=8$ measurement points, a normalized radius of $r=0.3$ (corresponding to a half opening angle of $16.7 \mathrm{deg}$ ), and the first and the last measurement distance at $x_{1, \mathcal{L}}=0.5 \mathrm{D}=63 \mathrm{~m}$ and $x_{5, \mathcal{L}}=1.5 \mathrm{D}=189 \mathrm{~m}$. The optimal scan trajectory is illustrated in Figure 2. The coherence and transfer function of the correlation model and from the simulation below using simulated lidar measurements are plotted in Figure 3.

\section{CONTROLLER DESIGN}

In this section, the baseline controller is briefly described. Then the lidar-assisted collective pitch feedforward controller is derived first for perfect and then for realistic wind preview.

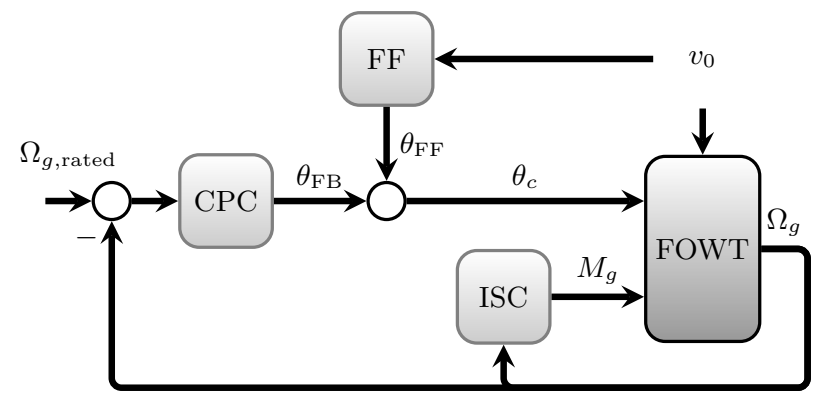

Figure 4: Feedback control loops and collective pitch feedforward controller assuming perfect wind preview.

\section{Baseline Feedback Controller}

The baseline feedback controller is an adaptation of the onshore baseline controller (Jonkman, 2007), which combines an indirect speed controller (ISC) for the generator torque and a collective blade pitch controller (CPC), see Figure 4. Both feedback controllers together adjust the turbine's operation under changing rotor-effective wind speed $v_{0}$ and use measurements of the generator speed $\Omega_{g}$. The generator speed signal is filtered using a single-pole low-pass filter, to mitigate high-frequency excitation of the control systems.

Below rated wind speed, the goal of the torque controller is to maximize the energy yield. Thus, the generator torque $M_{g}$ needs to be adjusted to track the inflow conditions. The generator torque is held constant above rated wind speed as long as the blade pitch angle remains above a threshold of $\theta_{\text {fine }}=1 \mathrm{deg}$.

The CPC regulates the rotor speed when the turbine operates at rated power. The collective blade pitch angle command $\theta_{c}$ is computed using a gain-scheduled PI controller on the speed error between the filtered and the rated generator speed $\Omega_{g \text {,rated. For }}$ the FOWT, the gains of the PI pitch controller are reduced to avoid negative damping of the platform pitch motion (Jonkman, 2007).

\section{Collective Pitch Feedforward Controller Design for Per- fect Wind Preview}

The basic idea of adding feedforward control to a feedback controller is to complete the two main tasks for controllers (reference signal tracking and disturbance compensation) independently by 


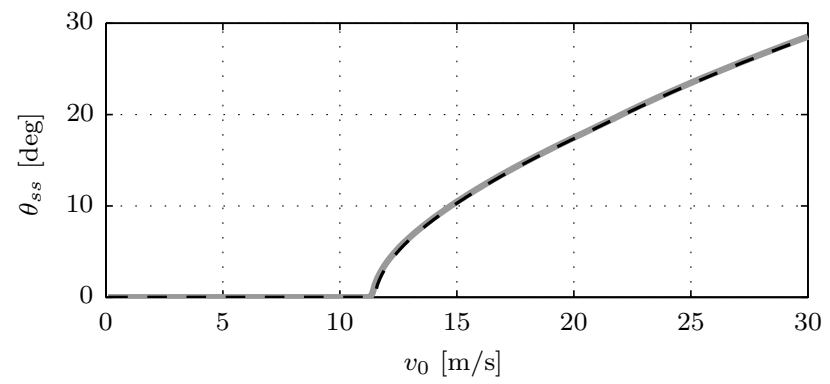

Figure 5: Steady pitch curve for the 5MW reference onshore (-) and floating (--) wind turbine obtained from steady-state simulations.

both controllers such that they do not interfere with each other.

For the design of the collective pitch feedforward controller $(\mathrm{FF})$, perfect knowledge of the rotor-effective wind speed $v_{0}$ is assumed in a first step. The feedforward controller is designed such that changes from the wind speed $v_{0}$ to the generator speed $\Omega_{g}$ are compensated by an additional blade pitch angle $\theta_{\mathrm{FF}}$. In this case, the feedforward controller is not counteracting the control action of CPC and ISC.

For controller design purposes, the rotor speed $\Omega$ is modeled by the following equation of motion:

$$
J \dot{\Omega}=M_{a}\left(v_{0}, \theta, q, \dot{q}\right)-M_{g} / i_{g b} .
$$

Here, $i_{g b}$ is the gearbox ratio and $J$ is the overall drive train moment of inertia about the rotation axis. Moreover, $M_{a}$ is the aerodynamic torque depending on the rotor-effective wind $v_{0}$, the collective pitch angle $\theta$, the vector of modes $q$, and its derivative $\dot{q}$.

The steady states for all turbine states and inputs depend in the closed loop on the steady wind speed $v_{0, s s}$. For above rated wind speed, the steady aerodynamic torque is constant:

$$
M_{a, s s}\left(v_{0, s s}, \theta_{s s}, q_{s s}, \dot{q}_{s s}\right)=M_{g, \text { rated }} / i_{g b} .
$$

Thus, $\theta, q$, and $\dot{q}$ need to be simultaneously aligned along their steady functions $\theta_{s s}, q_{s s}$, and $\dot{q}_{s s}$ to hold the rotor speed constant for changing $v_{0}$ in (6). These functions are obtained from steady state simulations. Figure 5 displays the steady collective pitch angle of the onshore and floating wind turbine as a function of $v_{0}$ with only minor differences.

With the availability of a wind preview, an inversion of the pitch actuator dynamics is possible, such that the collective blade pitch $\theta$ counteracts variations in $v_{0}$ based on a feedforward collective blade pitch command $\theta_{\mathrm{FF}}$. Here, the inverse pitch actuator dynamics is approximated by a simple preview time $\tau$. Aligning the modes and their derivatives along their steady functions is more complicated. However, the results of the simulations with perfect wind preview below show that aligning only the collective pitch angle already provides very good results compared to the feedback only case and that the modes quickly approach their steady values.

Similar to the feedforward controller for onshore wind turbines, a feedforward pitch rate $\dot{\theta}_{\mathrm{FF}}$ is added to the input of the integrator included in the feedback controller instead of adding the pitch angle $\theta_{\mathrm{FF}}$ to the output $\theta_{\mathrm{FB}}$ of the feedback controller: This modification simplifies the integration of anti-windup algorithms and switching the feedforward controller on and off.

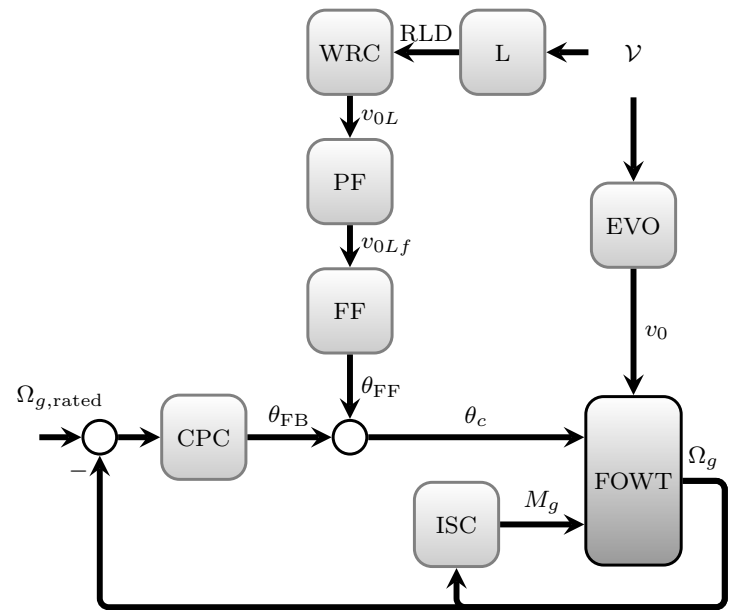

Figure 6: Feedback control loops and collective pitch feedforward controller assuming realistic wind preview.

\section{Collective Pitch Feedforward Controller Design for Re-} alistic Wind Preview

In reality, the rotor-effective wind speed $v_{0}$ cannot be measured perfectly. As depicted in Figure 6, the lidar system (L) is attempting to measure a three-dimensional wind field $\mathcal{V}$ and is only able to provide raw lidar data (RLD). In this investigation, the RLD are the line-of-sight wind speeds $v_{l o s}$, the index of the measurement point $i$ (1 to 8 ), and the time stamp of each measurement. The velocity $\left[\begin{array}{lll}\dot{x}_{L, \mathcal{W}} & \dot{y}_{L, \mathcal{W}} & \dot{z}_{L, \mathcal{W}}\end{array}\right]^{T}$, the pitch angle $\Theta_{L}$, and the roll angle $\Phi_{L}$ of the lidar system are added to the RLD. These values can be provided by an inertial measurement unit (IMU), which is already installed on some commercial lidar systems.

The RLD are transferred to the model-based wind field reconstruction (WRC) during the simulation. Similar to the approach for floating lidar systems (Schlipf et al., 2012), the pitch angle $\Theta_{L}$ and the roll angle $\Phi_{L}$ are used to transform the projected coordinates $\left[\begin{array}{lll}x_{i, \mathcal{L}} & y_{i, \mathcal{L}} & z_{i, \mathcal{L}}\end{array}\right]^{T}$ into the wind coordinate system $\mathcal{W}$, which is aligned with the mean wind direction and moves and rotates with the FOWT. Thus, measurements of the platform's translational DOFs are not necessary for the WRC. Based on (5), all line-of-sight wind speeds $v_{l o s, i}$ are translated into longitudinal wind speed components $u_{i, \mathcal{W}}$ by assuming point measurements and perfect alignment $\left(v_{i, \mathcal{W}}=w_{i, \mathcal{W}}=0\right)$ by

$$
u_{i, \mathcal{W}}=\frac{v_{l o s, i}+x_{n i, \mathcal{W}} \dot{x}_{L, \mathcal{W}}+y_{n i, \mathcal{W}} \dot{y}_{L, \mathcal{W}}+z_{n i, \mathcal{W}} \dot{z}_{L, \mathcal{W}}}{x_{n i, \mathcal{W}}},
$$

where $\left[\begin{array}{lll}x_{n i, \mathcal{W}} & y_{n i, \mathcal{W}} & z_{n i, \mathcal{W}}\end{array}\right]^{T}$ is the normalized laser vector calculated from the transformed coordinates similar to (2). All $u_{i, \mathcal{W}}$ are stored in a buffer and condensed to an estimate $v_{0 L}$ of the rotor-effective wind speed. Here, Taylor's Hypothesis is used for the wind evolution (EVO) and therefore measurements of the several distances can be combined by shifting them in time (Schlipf et al., 2014). The transfer function between $v_{0 L}$ and $v_{0}$ is known to be the optimal prefilter (PF) for the lidar estimate to remove all uncorrelated frequencies (Simley and Pao, 2013; Schlipf et al., 2013a). Here, a second-order low pass filter (Butterworth) with a cut-off frequency of $f_{\text {cutoff }}=0.201 \mathrm{~Hz}$ is fitted to the transfer function, see Figure 3. Since the filtered estimated $v_{0 L f}$ is close to the rotor-effective wind speed $v_{0}$, the collective pitch feedforward controller can also be applied using realistic wind preview. 
Table 1: Maximum values of the simulation with an EOG at $25 \mathrm{~m} / \mathrm{s}$ with perfect wind preview using the $5 \mathrm{MW}$ reference floating wind turbine (see Figure 7).

\begin{tabular}{l|rr|r} 
& $\mathrm{FB}$ & $\mathrm{FB}+\mathrm{FF}$ & $\frac{\mathrm{FB}+\mathrm{FF}}{\mathrm{FB}}[\%]$ \\
\hline$\Delta \Omega[\mathrm{rpm}]$ & 2.60 & 0.03 & 1.1 \\
$\Delta \Theta_{P}[\mathrm{deg}]$ & 0.345 & 0.022 & 6.3 \\
$M_{y T}[\mathrm{MNm}]$ & 55.9 & 34.8 & 62.2
\end{tabular}

\section{SIMULATION RESULTS}

In this section, the collective pitch feedforward controller is evaluated by simulations using perfect wind preview and simulated lidar measurements.

\section{Simulations Using Perfect Wind Preview}

In a first simulation study, the collective pitch feedforward controller is tested assuming perfect wind preview. For this purpose, the full aero-hydro-servo-elastic model is disturbed by an Extreme Operating Gust (EOG) at $25 \mathrm{~m} / \mathrm{s}$ according to the International Electrotechnical Commission (2005). The proposed feedforward controller can achieve almost perfect cancellation of the effect from $v_{0}$ to $\Omega$, see Figure 7 . The overshoot of the rotor speed (deviation from $\Omega_{\text {rated }}=12.1 \mathrm{rpm}$ ) can be reduced by $98.9 \%$, the maximum deviation from the static platform pitch angle $\Theta_{P}$ by $93.7 \%$, and the maximum tower base fore-aft bending moment $M_{y T}$ by $37.8 \%$ compared to the feedback controller, see Table 1 .

The proposed feedforward controller demonstrates a good robustness against model uncertainties. Although the controller is designed with a nonlinear model with only one DOF (rotor speed), static aerodynamics, and no hydrodynamics, it is able to almost perfectly cancel out the effect from the rotor-effective wind to the rotor speed for a full aero-hydro-servo-elastic model with 22 DOFs over a large range of wind speeds. This yields also less oscillation in the turbine and platform structure, such as the tower top displacement $x_{T}$ and the platform displacement $x_{P}$.

\section{Simulations Using Simulated Lidar Measurements}

In a second simulation study, the robustness against wind measurement errors is examined. For this investigation, a turbulent wind field with turbulence class "A" according to (International Electrotechnical Commission, 2005), resulting in a turbulence intensity of $17 \%$ and a length of over $1 \mathrm{~h}$ is generated with TurbSim with a mean wind speed of $\bar{u}=18 \mathrm{~m} / \mathrm{s}$. The width and height of the wind field are each $150 \mathrm{~m}$ and the resolution is $5 \mathrm{~m}$, resulting in $31 \times 31$ grid points. The temporal resolution is $0.25 \mathrm{~s}$. With this wind field, the full aero-hydro-servo-elastic model is simulated for $3630 \mathrm{~s}$. All states are initialized with their steady values corresponding to the rotor-effective wind speed (average over the rotor disk) at the beginning of the simulation. The first $30 \mathrm{~s}$ are ignored to avoid falsification of the results due to minor initialization effects. Again, calm sea is assumed. During simulations, the wind field is scanned by the lidar simulator taking into account the current position, velocity, and inclination of the lidar system. From the raw lidar data, the rotor-effective wind speed is reconstructed and filtered as described above.

Figure 8 illustrates a representative $5 \mathrm{~min}$ period of the simulation. In the top part of the figure a good agreement between the rotor-effective wind speed from the wind field and its filtered and time-shifted estimate based on the raw lidar data can be observed. With this more realistic wind preview, the variation
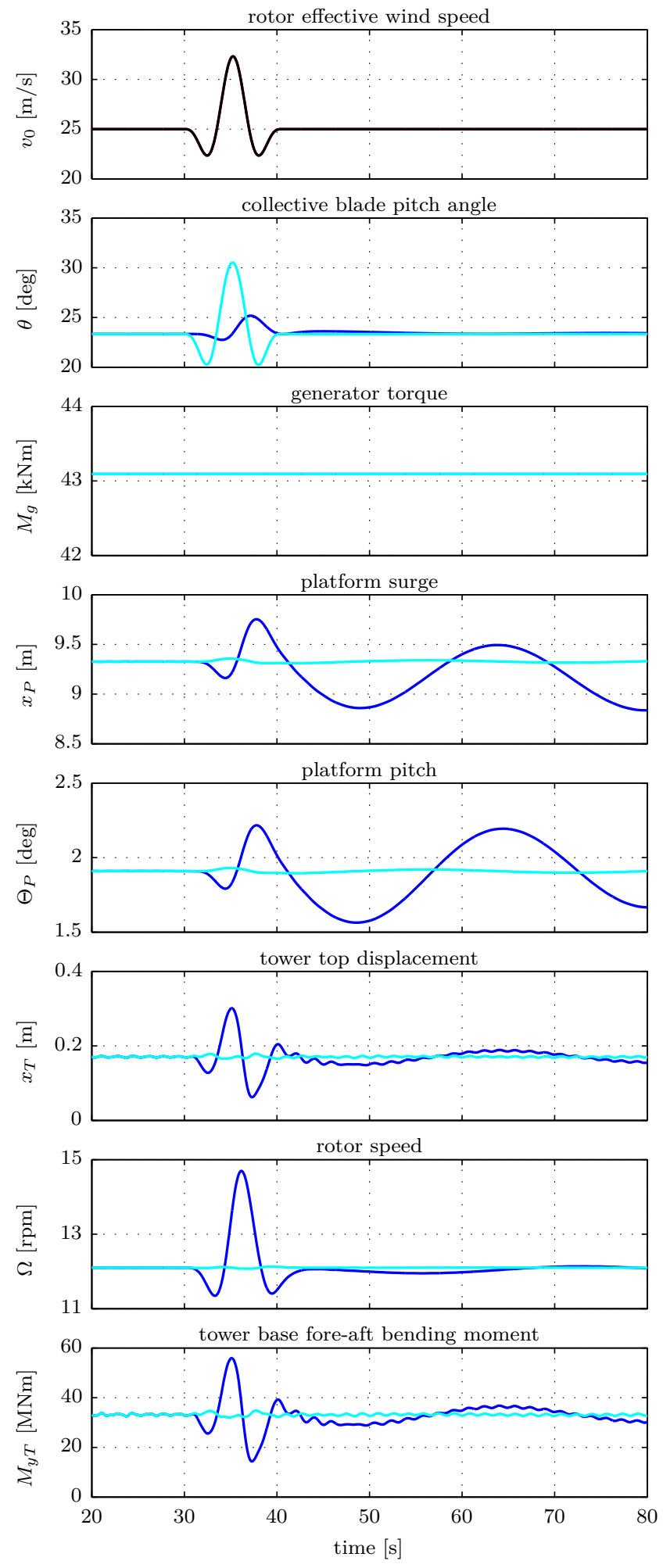

Figure 7: Reaction to an EOG at $25 \mathrm{~m} / \mathrm{s}$ with perfect wind preview (一) using the $5 \mathrm{MW}$ reference wind turbine. Feedback controller only (-) and with additional feedforward (-). 
Table 2: Comparison of the results for the $1 \mathrm{~h}$ simulation at $18 \mathrm{~m} / \mathrm{s}$ using feedback $(F B)$ and using feedback and feedforward control $(F B+F F)$.

\begin{tabular}{l|rr|r} 
& $\mathrm{FB}$ & $\mathrm{FB}+\mathrm{FF}$ & $\frac{\mathrm{FB}+\mathrm{FF}}{\mathrm{FB}}[\%]$ \\
\hline $\operatorname{DEL}\left(M_{y T}\right)[\mathrm{MNm}]$ & 139.9 & 111.9 & 80.0 \\
$\operatorname{DEL}\left(M_{L S S}\right)[\mathrm{MNm}]$ & 2.86 & 2.65 & 92.7 \\
$\operatorname{DEL}\left(M_{\text {oop } 1}\right)[\mathrm{MNm}]$ & 12.75 & 11.55 & 90.6 \\
$\operatorname{STD}(\Omega)[\mathrm{rpm}]$ & 0.952 & 0.180 & 18.9 \\
$\operatorname{STD}(\dot{\theta})[\mathrm{deg} / \mathrm{s}]$ & 0.253 & 0.368 & 145.8 \\
$\operatorname{STD}\left(x_{P}\right)[\mathrm{m}]$ & 2.599 & 2.150 & 82.7 \\
$\operatorname{STD}\left(\theta_{P}\right)[\mathrm{deg}]$ & 1.114 & 0.550 & 49.4 \\
$\operatorname{STD}\left(P_{e l}\right)[\mathrm{MW}]$ & 0.4018 & 0.0752 & 18.7 \\
$\operatorname{EP}[\mathrm{MWh}]$ & 5.0213 & 5.0020 & 99.615
\end{tabular}

in the rotor speed $\Omega$ is still reduced significantly. Additionally, the variation in platform displacement $x_{P}$, platform pitch $\Theta_{P}$, the tower top displacement $x_{T}$, and the resulting tower base fore-aft bending moment $M_{y T}$ are reduced. The effect of the collective pitch feedforward controller can be observed clearly in the Power Spectral Densities (PSDs) of Figure 9. The lidarassisted controller can significantly reduce the influence of the wind disturbance to rotor speed at low frequencies. Since the adaptive filter has a cut-off-frequency at $f_{\text {cutoff }}=0.201 \mathrm{~Hz}$, the improvement minimizes above this frequency and no reduction is achieved at the damped eigenfrequency of the tower $(0.5 \mathrm{~Hz})$ and the $3 P$ (three-times-per-revolution) frequency $(0.6 \mathrm{~Hz})$. In addition, the tower base fore-aft bending moment is significantly reduced for low frequencies, especially at the dominant platform pitch eigenfrequency of $0.03 \mathrm{~Hz}$. Since the steady-states of the tower and the collective pitch angle are changing with the mean wind speed, no reduction is possible at frequencies close to $0 \mathrm{~Hz}$. The reduction in rotor speed and power variation for low frequencies is achieved by a higher pitch activity, which is represented by the pitch rate $\dot{\theta}$.

Table 2 summarizes the results of the $1 \mathrm{~h}$ simulation at $18 \mathrm{~m} / \mathrm{s}$. For the calculation of the Damage Equivalent Loads (DELs), a reference number of cycles $n_{\text {ref }}=2 \times 10^{6}$ is used. Further, a Wöhler exponent of $m=4$ is assumed for the fatigue load calculation of the tower base fore-aft bending moment $M_{y T}$ and the low-speed shaft torque $M_{L S S}$. For $M_{o o p 1}$, the out-of-plane blade root bending moment of blade 1, a Wöhler exponent of $m=10$ is applied. Besides the above-mentioned load reduction on the tower base $(20 \%)$, additional load reduction on shaft and blade root ( 7 and $9 \%$, respectively) is achieved. This is mainly because the standard deviation (STD) of the platform pitch angle $\Theta_{P}$ can be reduced by $50 \%$. Over $80 \%$ reduction in the standard deviation of the rotor speed can be achieved. Since the generator torque is held constant for both controllers, this results in similar reduction in the standard deviation of the electrical power $P_{e l}$. When the feedforward controller is enabled, the rated power of $5 \mathrm{MW}$ is better tracked and the energy production (EP) of the $1 \mathrm{~h}$ simulation is closer to $5 \mathrm{MW} \times 1 \mathrm{~h}$. The relative increase of $46 \%$ in the standard deviation of the pitch rate is significant, but the absolute value $(0.368 \mathrm{deg} / \mathrm{s})$ is still below the value $(0.800 \mathrm{deg} / \mathrm{s})$ of the onshore reference wind turbine controlled by the onshore feedback controller with the same wind field.

These results confirm that collective pitch feedforward control is very promising for FOWTs even considering wind preview measurement uncertainties. The next subsection will rank the improvements in rotor speed regulation.
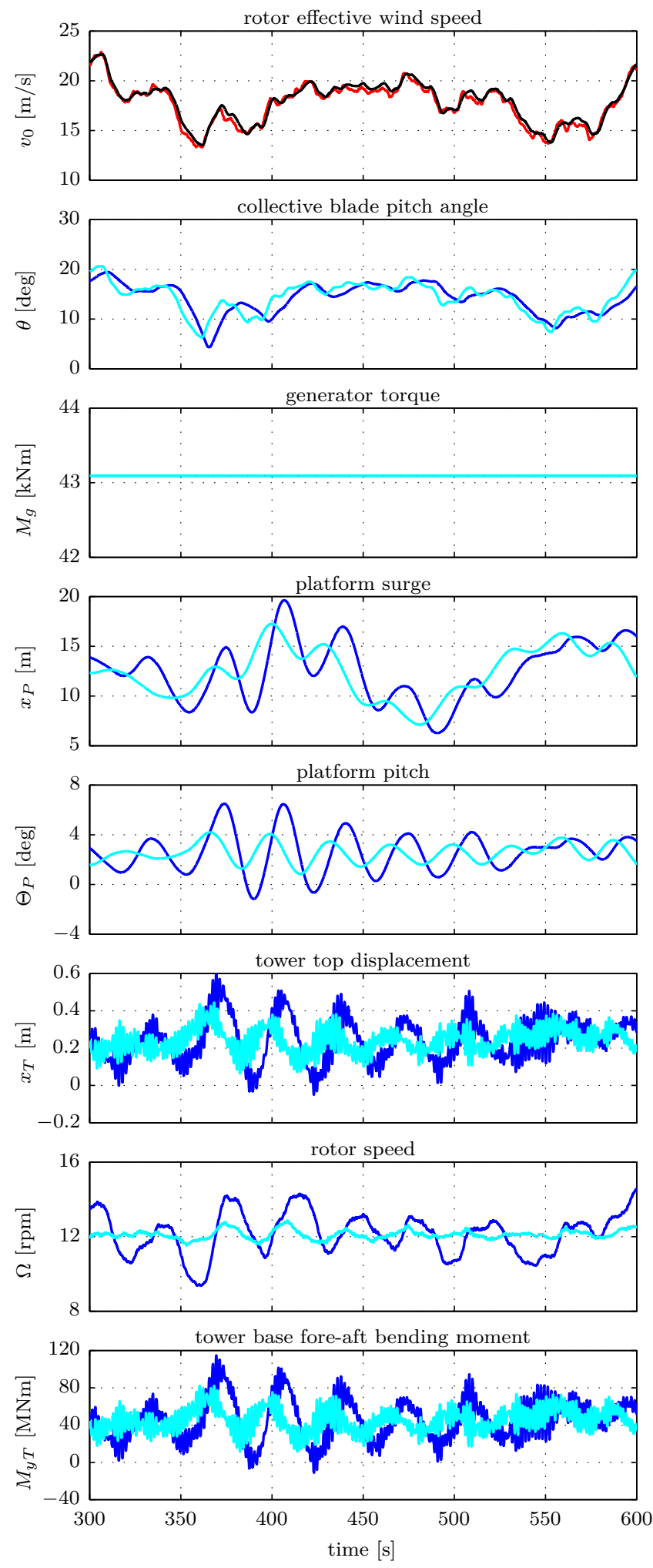

Figure 8: Reaction to a turbulent wind field with mean wind speed of $18 \mathrm{~m} / \mathrm{s}$ (illustrative $5 \mathrm{~min}$ excerpt). Top: rotor-effective wind speed (-) and its filtered lidar estimate (-). Rest: Feedback controller only (-) and with additional feedforward (-) using simulated lidar measurements. 

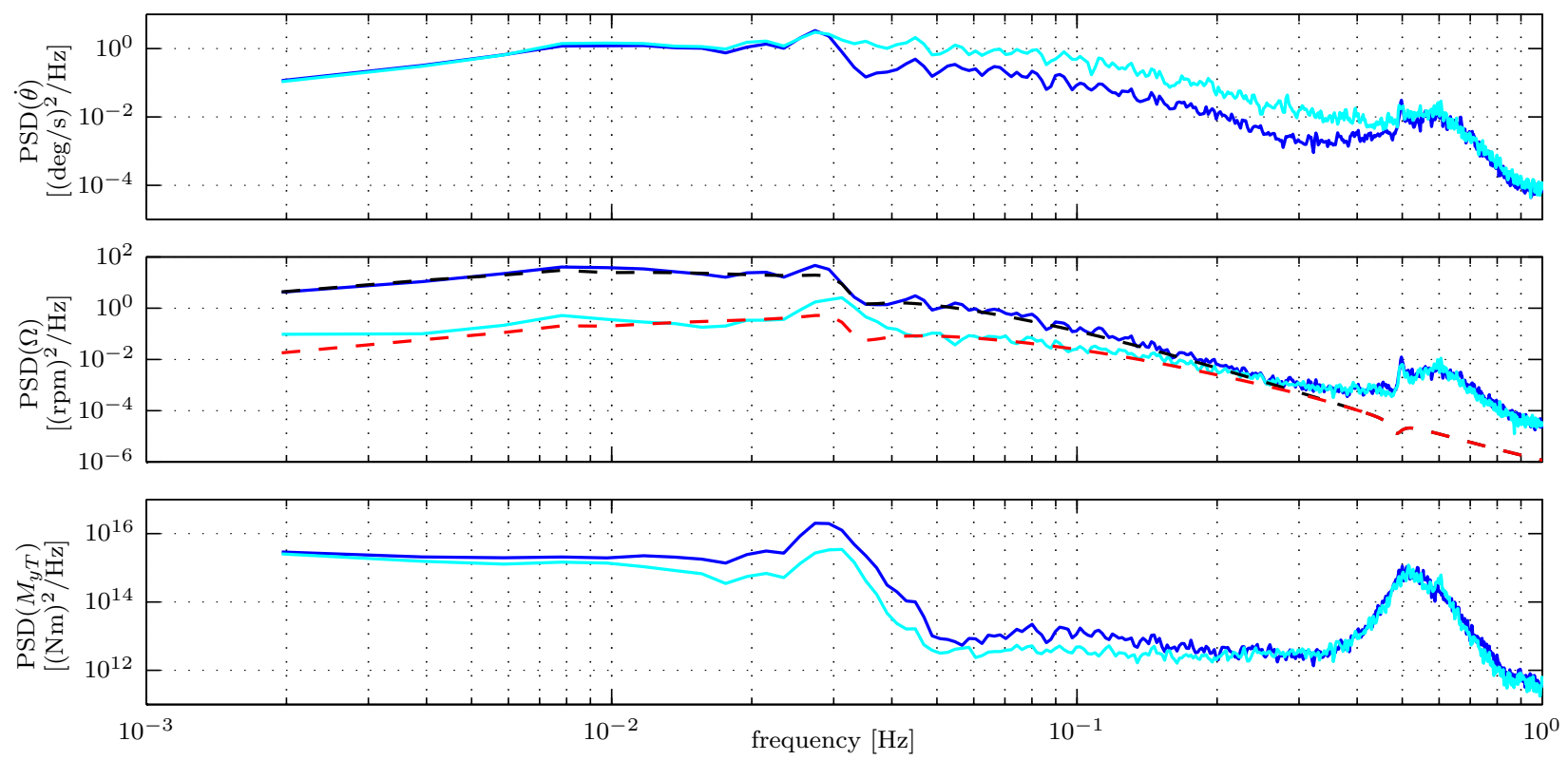

Figure 9: PSD for the $1 \mathrm{~h}$ simulation at $18 \mathrm{~m} / \mathrm{s}$ : Feedback controller only (一) and with additional feedforward (-). Theoretical rotor speed spectrum for the feedback controller only (--) and with additional feedforward (--).

\section{Evaluation of the Results}

The PSD of the rotor speed is compared to the theoretical minimum PSD which can be achieved by a perfect feedforward controller assuming linear turbine dynamics.

The linear model around the mean wind speed of $18 \mathrm{~m} / \mathrm{s}$ is obtained from the FAST model and the tools provided by Jonkman and Buhl (2005) enabling only the following four DOFs: platform surge $x_{P}$, platform pitch $\Theta_{P}$, tower top displacement $x_{T}$, and rotor/generator speed $\Omega$, as suggested by Sandner et al. (2014).

Using the linearized turbine model for the specific operating point, the rotor speed can be written as a function of $v_{0}$ and the reconstructed lidar measurement $v_{0 L}$ :

$$
\Omega=G_{\Omega v_{0}} v_{0}+G_{\Omega \theta} G_{\mathrm{FF}} v_{0 L}
$$

where $G_{\Omega v_{0}}$ and $G_{\Omega \theta}$ are the closed-loop transfer functions from $v_{0}$ to $\Omega$ and $\theta$ to $\Omega$, respectively, and $G_{\mathrm{FF}}$ is the transfer function of the feedforward controller. The closed-loop transfer functions include the linearized FAST model, the pitch actuator, the generator speed filter, and the gain-scheduled PI controller.

Under the assumption that the closed-loop dynamics of the turbine/controller pair are linear and that $v_{0}$ and $v_{0 L}$ are jointly wide-sense stationary random processes, the feedforward controller that minimizes the PSD of $\Omega$ is defined by the frequency response

$$
G_{\mathrm{FF}}=-G_{\Omega \theta}^{-1} G_{\Omega v_{0}} \frac{S_{R L}}{S_{L L}},
$$

where $S_{R L}$ is the cross-power spectral density between the rotoreffective wind speed $v_{0}$ and the lidar measurement $v_{0 L}$, and $S_{L L}$ is the PSD of the lidar measurement (Simley and Pao, 2013). The transfer function in Equation (10) is comprised of two stages. First, the transfer function $-G_{\Omega \theta}^{-1} G_{\Omega v_{0}}$ is the ideal feedforward controller that reduces the rotor speed error to zero if $v_{0 L}=v_{0}$. Second, because the lidar does not provide a perfect measurement of $v_{0}$, the filter $S_{R L} / S_{L L}$ yields the minimum mean square error (MMSE) linear estimate of $v_{0}$ based on $v_{0 L}$ (Kailath et al., 2000).
Note that $S_{R L} / S_{L L}$ is equivalent to the transfer function $G_{R L}$ plotted in Figure 3. However, since the wind speed signals $v_{0}$ and $v_{0 L}$ are modeled as jointly Gaussian random processes, the filter $S_{R L} / S_{L L}$ is not only the MMSE linear estimator of $v_{0}$, but the optimal MMSE estimator of $v_{0}$ among all possible estimators (Kailath et al., 2000). By using a MMSE filter to estimate $v_{0}$ from $v_{0 L}$ prior to the ideal feedforward controller stage, the controller (10) also minimizes the PSD of $\Omega$ (Simley and Pao, 2013).

As explained in (Simley and Pao, 2013), the PSD of the regulated output $\Omega$ described by Equation (9) when using the optimal feedforward controller defined in Equation (10) is given by

$$
S_{\Omega \Omega}=\left|G_{\Omega v_{0}}\right|^{2} S_{R R}\left(1-\gamma_{R L}^{2}\right)
$$

with $\gamma_{R L}^{2}$ representing the coherence between the reconstructed lidar measurement and the rotor-effective wind speed. The optimal achievable rotor speed spectra calculated using Equation (11) with feedback only (equivalent to setting $\gamma_{R L}^{2}=0$ (Simley and Pao, 2013)) and with optimal feedforward control are compared with the corresponding spectra from simulation in Figure 9. The coherence $\gamma_{R L}^{2}$, shown in Figure 3, and the PSD $S_{R R}$ are calculated using the analytic model presented in (Schlipf et al., 2013a).

As can be seen in Figure 9, the theoretical rotor speed spectrum with feedback only and the theoretically optimal spectrum with feedforward control do not perfectly match the results from simulation. For example, the dynamics of the linearized model at the tower eigenfrequency near $0.5 \mathrm{~Hz}$ do not match the simulated dynamics, and the linearized turbine model does not include dynamics associated with the 3P frequency $(0.6 \mathrm{~Hz})$ caused by three rotating blades. Furthermore, as exhibited in Figure 8, the turbine deviates significantly from the linearization operating point of $18 \mathrm{~m} / \mathrm{s}$, and thus the linear model could be inaccurate for significant portions of the simulation. Nevertheless, the implemented feedforward control strategy achieves close to the minimum possible rotor speed spectrum obtained using the simple linear model, up to the adaptive filter cut-off-frequency. 


\section{CONCLUSIONS AND OUTLOOK}

In this work a collective pitch feedforward controller for a reference floating wind turbine is designed and evaluated. The feedforward controller is derived from a reduced nonlinear model of the wind turbine and can be combined with a baseline feedback controller and adequate lidar data processing.

In a first simulation study the proposed controller proves its robustness against model uncertainties: Assuming perfect wind preview, the combined feedforward-feedback controller is able to almost perfectly cancel out the effect from the rotor-effective wind to the rotor speed for a full aero-hydro-servo-elastic simulation model. In a second simulation study, the controller performance in the presence of wind measurement uncertainties is investigated. The wind preview is based on a detailed simulation of a nacellebased lidar system moving with the floating wind turbine. The raw lidar data are condensed during the simulation with a turbulent wind field to an estimate of the rotor-effective wind speed by model-based wind field reconstruction and filtered by an adaptive filter depending on the mean wind speed. Under these more realistic conditions, the combined feedforward-feedback controller is compared to the feedback controller alone. Rotor speed and power variations are reduced by over $80 \%$. Additional load reduction on the tower, rotor shaft, and blades of 20,7 , and $9 \%$ is achieved, respectively. All reduction is mainly obtained at low frequencies. The improvements in rotor speed regulation are compared to the theoretical optimal results which can be achieved with the used lidar setup under theoretically optimal conditions. The proposed feedforward controller achieves results close to the optimum in terms of rotor speed regulation.

In future work, a more detailed fatigue analysis based on the design load case 1.2 from (International Electrotechnical Commission, 2005) with irregular waves will be performed to estimate the benefit of the proposed controller over the lifetime of a floating offshore wind turbine. Additional investigations are planned to transform the large reduction in rotor speed and power variations into further reduction of structural loads and increase in energy production. Furthermore, a comparison to feedback controllers with disturbance estimation such as the Disturbance Accommodation Control (DAC) without the need of preview wind measurements will be carried out.

\section{ACKNOWLEDGEMENTS}

The research leading to these results has received funding from the foundation "Karl Schlecht Stiftung (KSG)". Partial funding support from NREL is also gratefully acknowledged.

\section{REFERENCES}

Cariou, JP (2013). "Pulsed lidars," in Remote Sensing for Wind Energy, DTU Wind Energy-E-Report-0029(EN), pages 104-121. Fischer, B (2012). "Reducing rotor speed variations of floating wind turbines by compensation of non-minimum phase zeros," in Proceedings of the European Wind Energy Association Annual Event, Copenhagen, Denmark.

Fleming, P, Pineda, I, Rossetti, M, Wright, A, and Arora, D (2014). "Evaluating methods for control of an offshore floating turbine," in Proceedings of the 33rd International Conference on Ocean, Offshore and Arctic Engineering, San Francisco, USA.

International Electrotechnical Commission (2005). "Wind turbines - Part 1: Design requirements," International standard, IEC 61400-1, third edition.

Jonkman, BJ (2009). "TurbSim user's guide," Technical Report TP-500-46198, NREL.
Jonkman, J (2007). "Dynamics modeling and loads analysis of an offshore floating wind turbine," Technical Report NREL/TP$500-41958$

Jonkman, J and Buhl, ML (2005). "FAST user's guide," Technical Report EL-500-38230, NREL.

Kailath, T, Sayed, AH, and Hassibi, B (2000). Linear Estimation, Prentice Hall, Upper Saddle River, NJ.

Lackner, MA and Rotea, MA (2011). "Structural control of floating wind turbines," Mechatronics, volume 21(4), pages $704-$ 719

Larsen, TJ and Hanson, TD (2007). "A method to avoid negative damped low frequent tower vibrations for a floating, pitch controlled wind turbine," Journal of Physics: Conference Series, volume 75 , page 012073 .

Namik, H and Stol, K (2010). "Individual blade pitch control of a floating offshore wind turbine on a tension leg platform," in Proceedings of the 48th AIAA Aerospace Sciences Meeting Including the New Horizons Forum and Aerospace Exposition, Orlando, USA

Raach, S, Schlipf, D, Sandner, F, Matha, D, and Cheng, PW (2014). "Nonlinear model predictive control of floating wind turbines with individual pitch control," in Proceedings of the American Control Conference, Portland, Oregon, USA.

Sandner, F, Schlipf, D, Matha, D, and Cheng, PW (2014). "Integrated optimization of floating wind turbine systems," in Proceedings of the 33rd International Conference on Ocean, Offshore and Arctic Engineering, San Francisco, USA.

Schlipf, D, Fleming, P, Haizmann, F, Scholbrock, A, Hofsäß, M, Wright, A, and Cheng, PW (2014). "Field testing of feedforward collective pitch control on the CART2 using a nacelle-based lidar scanner," Journal of Physics: Conference Series, volume 555(1), page 012090 .

Schlipf, D, Mann, J, and Cheng, PW (2013a). "Model of the correlation between lidar systems and wind turbines for lidar assisted control," Journal of Atmospheric and Oceanic Technology, volume 30(10), pages 2233-2240.

Schlipf, D, Rettenmeier, A, Haizmann, F, Hofsäß, M, Courtney, M, and Cheng, PW (2012). "Model based wind vector field reconstruction from lidar data," in Proceedings of the German Wind Energy Conference DEWEK, Bremen, Germany.

Schlipf, D, Sandner, F, Raach, S, Matha, D, and Cheng, PW (2013b). "Nonlinear model predictive control of floating wind turbines," in Proceedings of The 23rd International Offshore and Polar Engineering Conf, Anchorage, USA, ISOPE, Vol 1.

Schlipf, D, Trujillo, JJ, Basterra, V, and Kühn, M (2009). "Development of a wind turbine LIDAR simulator," in Proceedings of the European Wind Energy Conference, Marseille, France.

Scholbrock, A, Fleming, P, Fingersh, L, Wright, A, Schlipf, D, Haizmann, F, and Belen, F (2013). "Field testing LIDAR based feed-forward controls on the NREL controls advanced research turbine," in Proceedings of the 51st AIAA Aerospace Sciences Meeting Including the New Horizons Forum and Aerospace Exposition, Dallas, USA.

Simley, E and Pao, LY (2013). "Reducing lidar wind speed measurement error with optimal filtering," in Proceedings of the American Control Conference, Washington D.C., USA.

Taylor, GI (1938). "The spectrum of turbulence," Proceedings of the Royal Society of London. Series A - Mathematical and Physical Sciences, volume 164(919), pages 476-490.

van der Veen, G, Couchman, I, and Bowyer, R (2012). "Control of floating wind turbines," in Proceedings of the American Control Conference, Montreal, Canada. 